\title{
Géolinguistique
}

15 | 2015

La géographie linguistique au Brésil

\section{Pour une approche géo-sociolinguistique de la réalité linguistique brésilienne}

For a Geo-Sociolinguistic Approach to the Brazilian Linguistic Reality

\section{Silvia Figueiredo Brandão}

\section{(2) OpenEdition}

Journals

Édition électronique

URL : http://journals.openedition.org/geolinguistique/666

DOI : 10.4000/geolinguistique.666

ISSN : 2650-8176

Éditeur

UGA Éditions/Université Grenoble Alpes

\section{Édition imprimée}

Date de publication : 1 décembre 2015

Pagination : 191-214

ISBN : 978-2-84310-317-9

ISSN : 0761-9081

\section{Référence électronique}

Silvia Figueiredo Brandão, « Pour une approche géo-sociolinguistique de la réalité linguistique brésilienne ", Géolinguistique [En ligne], 15 | 2015, mis en ligne le 15 février 2019, consulté le 30 octobre 2020. URL : http://journals.openedition.org/geolinguistique/666 ; DOI : https://doi.org/10.4000/ geolinguistique.666 


\title{
Pour une approche géo-sociolinguistique de la réalité linguistique brésilienne
}

\author{
Silvia Figueiredo Brandão \\ Universidade Federal do Rio de Janeiro - CNPq - FAPERJ (Brésil)
}

\section{Résumé}

Cet article traite de l'importance d'allier les principes et méthodes de la géolinguistique et de la sociolinguistique pour mieux caractériser la réalité linguistique brésilienne, hétérogène et polarisée. Cette diversité est due au processus de colonisation du pays, dans lequel se sont confrontés des groupes ethniques de différentes origines, et aussi à cause de changements significatifs vécus par la société brésilienne, surtout à partir du milieu du $\mathrm{Xx}^{\mathrm{e}}$ siècle, quand il y a eu une migration intensive des zones rurales vers les grands centres urbains.

Nous tentons de démontrer que la géolinguistique, malgré sa longévité, s'est efforcée de se renouveler, surtout dans sa version pluridimensionnelle. Cette version intègre certaines des procédures méthodologiques de la sociolinguistique afin de contribuer à une meilleure caractérisation des motivations structurelles et sociales qui déterminent l'implémentation de variantes et entraînent les processus de changement.

\section{Mots-clés}

Géolinguistique, Brésil, sociolinguistique.

\section{Abstract}

This article discusses the importance of allying the principles and methods of Geolinguistics and Sociolinguistics in order better to characterise the realities of language in Brazil, where it is heterogeneous and polarised as a result of not only the process of colonisation, which brought ethnic groups 
of widely differing origins into contact, but also significant changes in Brazilian society, particularly during the latter half of the twentieth century, when there was intensive migration from rural areas into large towns.

The intention is to demonstrate that, despite its longevity, Geolinguistics has endeavoured to renew itself, especially in its multidimensional version, which incorporates some of the methodological procedures of Sociolinguistics, with a view to characterising better the structural and social factors that determine the implementation of variants and steer processes of change.

\section{Keywords}

Geolinguistic, Brazil, Sociolinguistic.

Le débat sur la pertinence à mener des études au sein de la géographie linguistique dans les pays dits du Nouveau Monde est encore d'actualité, ces derniers présentant une complexité socioculturelle que la méthode développée par Gilliéron ne visait pas. Conçu comme un moyen d'enregistrer, dans un domaine géographique donné, les formes les plus conservatrices des langues et, d'une certaine façon, de prouver, en réaction à la proposition néogrammairienne, que chaque mot a son histoire, la méthode est arrivée aux Amériques, directement ou indirectement, sous l'influence des travaux de dialectologues européens réputés.

Jacob Jud, par exemple, était aux États-Unis pour contribuer à l'élaboration d'un atlas linguistique des États-Unis et du Canada, qui a abouti au Linguistic Atlas of New England (LANE), dirigé par Hans Kurath (19391943). Au moment où les premiers résultats du LANE ont commencé à apparaître, certaines voix se sont levées pour attirer l'attention sur la faible représentativité régionale et surtout sociale des matériaux enregistrés, même si Kurath avait été, selon les mots de Joseph Allen, «le premier dialectologue qui a pris en compte des facteurs sociaux dans un projet où l'on enquêtait systématiquement sur un groupe de population» (Fernández, 1991, p. 101) ${ }^{1}$.

1. Toujours d'après Fernández (1991, p. 101), Kurath n'a utilisé que $22 \%$ des femmes et $1,2 \%$ des participants de moins de 30 ans, mais a distingué trois niveaux socioculturels qui, combinés avec l'âge, ont été réduits à deux : type $\mathrm{A}$ : personnes âgées ou considérées comme «vivant à l'ancienne»; type B : personnes d'âge moyen ou considérées comme plus modernes. 
Parmi les voix qui ont examiné de façon critique la portée de la géographie linguistique, la sociologue Glenna Pickford a affirmé que les atlas linguistiques n'étaient pas «au plus haut niveau de la recherche scientifique » (1956, p. 229), en raison des techniques de collecte de données et des critères de sélection d'informateurs, qui manqueraient, respectivement, de validité et de fiabilité. Même avant l'apparition de la sociolinguistique d'inspiration labovienne, Pickford attirait l'attention sur le besoin de représenter les différents segments de la société pour un enregistrement fiable de la dynamique linguistique dans une zone donnée. À ce propos, il faut mentionner Tomás Navarro Tomás, pour qui les langues européennes apportées aux Amériques finiraient par générer une géographie linguistique spécifiquement américaine, en raison des particularités de ce territoire, où il y a non seulement de grandes villes mais aussi de vastes zones vides et où on observe un flux continu de migrations (Mouton, 2003). Comme l'a noté Brandão, il y avait d'autres défis que ceux mentionnés ci-dessus :
Ainsi que l'ont montré les chercheurs qui se consacrent, à l'heure actuelle, à des études de nature géolinguistique, dans des pays comme le Brésil, il faut prendre également en compte la complexité sociolinguistique qui caracté- rise les pays de l'Amérique, comme ont essayé de le faire les créateurs du LANE et, plus récemment, ceux de l'Atlas Lingüístico Diatópico y Dias- trático del Uruguay - ADDU (Thun \& Elizaincín, 2000) ainsi que ceux de l'Atlas Lingüístico do Brasil - ALiB, projet encore en cours. (2013, p. 2)

La géographie linguistique a de nombreux mérites, y compris celui d'avoir inauguré la pratique de la recherche sur le terrain, de l'organisation de corpora, de l'observation de la langue dans sa dynamique, présentant un aperçu global de la variation sur un territoire particulier, donnant lieu à des inférences de nature socio-historique, attirant l'attention sur des questions concernant les limites dialectales, les processus de changement, la propagation de formes et de sons. La sociolinguistique, à son tour, essaie de connaître en profondeur les variables linguistiques qui sont pertinentes dans les communautés de parole, permettant de vérifier en détail les motivations structurelles et sociales qui déterminent l'accomplissement des variantes et des processus de changement. Ainsi, dans des pays comme le Brésil, d'une grande complexité sociale, il semble pertinent de combiner les nombreuses contributions de la dialectologie pratiquée aujourd'hui d'après de nouvelles bases aux principes théoriques et méthodologiques de la sociolinguistique variationnelle, pour l'analyse de phénomènes variables qui méritent d'être observés avec plus de précision.

Comme on considère les pratiques méthodologiques de la géolinguistique et de la sociolinguistique complémentaires, cet article a pour objectif 
de montrer l'importance de développer des études de nature géo-sociolinguistique afin de mieux caractériser le portugais du Brésil (désormais PB). À cette fin, dans la section suivante, nous présentons brièvement les principales hypothèses sur les origines du $\mathrm{PB}$, ainsi que certaines de ses caractéristiques; ensuite, seront présentés quelques aspects de la société brésilienne actuelle. Enfin, avant les considérations finales, une section discutera de la proposition de combiner les deux perspectives analytiques.

\section{1. À propos des origines et de quelques caractéristiques du PB}

Bien qu'il y ait eu au début du $\mathrm{Xx}^{\mathrm{e}}$ siècle des travaux qui cherchaient à focaliser, d'après une perspective dialectologique naissante, quelques-unes des caractéristiques des dialectes brésiliens, tels que ceux, entre autres, de Amaral (1986 [1920]) et de Nascentes (1953 [1922]), c'est seulement depuis les années 1970, lorsque la sociolinguistique d'inspiration labovienne est introduite dans le milieu universitaire brésilien, que l'on a commencé à développer des études systématiques sur certaines variables linguistiques typiques du PB. Ces études, au fil du temps, ne révélaient pas seulement une riche diversité, mais démontraient aussi que cette variété différait bien du portugais européen. En outre, la distance entre les variétés populaires et cultivées commençait à être claire aussi, fruit du processus de colonisation du pays. Comme une conséquence de ce l'on observait, sont apparus des débats sur les origines du $\mathrm{PB}$, débats déjà esquissés dans l'œuvre, entre autres, de Serafim da Silva Neto (1986 [1950]), selon une perspective plus proprement philologique. Les hypothèses sur les origines du PB populaire peuvent être assemblées en deux lignes interprétatives, comme l'indique Brandão (2015, p. 42). La première attache les caractéristiques du $\mathrm{PB}$ au massif contact multilinguistique et multiethnique qui s'est instauré au Brésil pendant la phase de la colonisation : dans sa version la plus forte, elle implique un processus de créolisation/décréolisation, tel que le proposent Guy $(1981,1988)$ et Holms (2003); dans sa version allégée, elle présuppose un processus de transmission linguistique irrégulière (Lucchesi, 2003, 2012). La deuxième ligne (Naro \& Scherre, 2007) postule que des faits tels que la réduction des flexions, l'élimination de la coda syllabique, la dénasalisation et d'autres processus qui ont lieu à la formation de pidgins et créoles, étaient déjà prévus dans la dérive des langues romanes et ont été mis en place en raison du dit contact multilinguistique. Comme le note Brandão :

Sabe-se que os portugueses, quando do início da colonização, já encontraram uma língua geral de base tupinambá adotada pelos índios da costa 
- pela qual optaram para fins de intercomunicação e catequese-, mais tarde denominada de língua geral paulista, cujo uso perduraria até o início do século XVIII. Também, no norte do país, havia a chamada língua geral amazônica ou nheengatu, ainda hoje falada em São Gabriel da Cachoeira, no Alto Rio Negro, Amazonas (Nobre, 2011). No entanto, apesar do significativo contingente de africanos importados para servirem como escravos e que, em alguns momentos, ultrapassava em grande escala o número de homens brancos, não se tem registro cabal de pidgins ou crioulos de base portuguesa utilizados pelas diferentes etnias que aqui chegaram e que pudessem justificar as características encontradas sobretudo na variedade popular do Português do Brasil, em especial no que respeita à concordância nominal e verbal. Servem de apoio para justificar a possível existência de tal(is) língua(s), características encontradas em comunidades afrodescendentes isoladas, em geral remanescentes de antigos quilombos (como em Helvécia, por exemplo), caracterizadas por Lucchesi (2012, p. 81) como "sítios arqueológicos da história sociolinguística do Brasil”².(2015, p. 42)

La situation sociolinguistique brésilienne est assez complexe. Aux niveaux syntaxique et morphosyntaxique, on observe des tendances similaires dans différentes régions du pays, par exemple, en ce qui concerne l'ordre des clitiques, les stratégies de relativisation, de remplissage du sujet et l'objet nul. L'incidence plus ou moins grande de marques de pluriel dans les constituants de la phrase nominale et dans le verbe est fortement affectée par le niveau d'éducation et, dans les grands centres urbains, la performance de ceux qui appliquent moins les marques de nombre pluriel est évaluée négativement. Sans doute, comme le soulignent Radtke et Thun, «stimuler

2. «On sait que les Portugais, au début de la colonisation, ont déjà trouvé une langue générale, de base tupinambá, adoptée par les indiens de la côte - laquelle a été choisie pour des buts communicatifs et de catéchèse - , plus tard appelée langue générale de São Paulo, dont l'usage allait durer jusqu'au début du XVIII ${ }^{\mathrm{e}}$ siècle. Au nord du pays, aussi, il y avait la dite langue générale amazonique ou nheengatu, parlée aujourd'hui encore à São Gabriel da Cachoeira, dans le Haut Rio Negro, Amazonas (Nobre, 2011). Cependant, malgré le contingent africain significatif importé pour servir d'esclaves et qui, parfois, dépassait à grande échelle le nombre d'hommes blancs, il n'y a pas d'enregistrement catégorique de pidgins ou de créoles de base portugaise utilisés par les différentes ethnies qui sont arrivées ici et qui pourraient justifier les caractéristiques trouvées surtout dans la variété populaire du portugais du Brésil, en particulier par rapport à l'accord nominal et verbal. Servent de support pour justifier l'existence éventuelle d'une telle(s) langue(s) des caractéristiques qui se retrouvent dans des communautés isolées d'ascendance africaine, en général des vestiges d'anciens quilombos (comme à Helvécia, par exemple), caractérisées par Lucchesi (2012, p. 81) comme "des sites archéologiques de l'histoire sociolinguistique du Brésil”.» 
des configurations syntaxiques de façon comparable et, en outre, dans des conditions de plus grande spontanéité possible est assez difficile» (1991, p.45), raison pour laquelle, d'après eux, la syntaxe reste seulement indirectement représentée dans la géolinguistique. D'autre part, l'usage des pronoms tu et você, de deuxième personne, en plus de présenter des contraintes stylistiques et sociales, sont soumis à des restrictions de nature diatopique : dans la région Sud du Brésil, par exemple, tandis que tu prédomine au Rio Grande do Sul et, surtout, à Santa Catarina, au Paraná, la forme você est presque catégorique (Coelho et al., 2015, p. 91). Quant à la variation phonétique et phonologique, ce sont les niveaux plus ou moins élevés de fréquence et non pas la présence ou l'absence catégorique de certaines variantes qui contribuent à la définition/classification des zones linguistiques. Peut-être que les variables les plus sensibles à des restrictions de nature diatopique, en ce qui concerne la distribution des variantes, sont les voyelles moyennes dans le contexte prétonique, le /R/ et le /S/ en coda syllabique et aussi $/ \mathrm{t} \mathrm{d} /$ dont la forme de réalisation devant [i] peut être dentale, palatalisée et affriquée. Ce ne fut pas par hasard qu'Antenor Nascentes (1953), en présentant sa proposition de la division du Brésil en deux grandes aires linguistiques - celle des parlers du Nord et celle des parlers du Sud -, l'a basée sur l'incidence plus élevée de variantes ouvertes dans la première de ces zones, et de variantes fermées dans la seconde. Des études sociolinguistiques montrent que ces variantes concurrencent également avec [i u] en raison d'une harmonisation vocalique ou d'un processus de réduction qui atteint quelques unités lexicales.

Pour donner une idée de la distribution, dans le contexte prétonique, des voyelles moyennes dans les parlers brésiliens, la figure 1 présente des données dans les parlers de 25 capitales des États brésiliens, ayant comme base l'étude de Mota et Cardoso (2015).

Laissées de côté les variantes hautes, on peut observer à travers la carte que les variantes [e o] prédominent dans tous les domaines, sauf dans le Nord-Est, où $[\varepsilon \rho]$ atteignent $60,7 \%$ des cas. Les variantes [ $[\varepsilon$ ] sont aussi considérables dans le Nord, avec un pourcentage de $36 \%$. On constate que l'incidence des variantes mi-fermées, compte tenu du continuum Sud $\rightarrow$ Nord (voir fig. 2), perd sa force, ce qui rend plus évidente la spécificité de la région Nord-Est. 


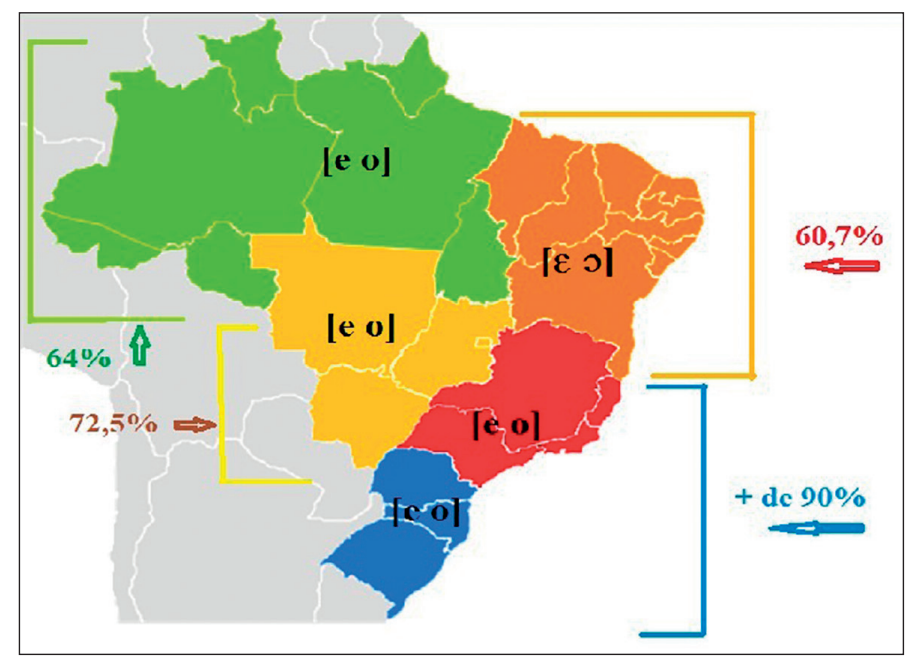

Figure 1. - Variantes moyennes de /e/ et de /o/ en contexte prétonique, prises ensemble, dans les parlers de 25 capitales, d'après des données du projet ALiB (Mota \& Cardoso, 2015).

Source : Callou \& Brandão (2013, inédit).

\begin{tabular}{|cccc|}
\hline SUD ET SUD-EST & CENTRE-OUEST & NORD & NORD-EST \\
$90 \%$ & $72,5 \%$ & $64 \%$ & $39,3 \%$ \\
& & & \\
\hline
\end{tabular}

Figure 2. - Fréquence des variantes [e o] en contexte prétonique dans les parlers de cinq régions géopolitiques brésiliennes (d'après Mota \& Cardoso, 2015).

Source : Callou \& Brandão (2013, inédit).

Un travail accompli par Rocha et Brandão (2014, inédit) fondé sur des études de Celia (2004), Almeida (2008), Dias (2008), Silveira (2008), Carmo (2009), Yacovenco (1993) et Rocha (2013), tous guidés par les principes théoriques et méthodologiques de la sociolinguistique quantitative, montre que, tandis que dans le Sud-Est il n'y a que $10 \%$ de voyelles moyennes ouvertes, il existe des zones qui se distinguent par l'occurrence de ces variantes (voir fig. 3). Rocha et Brandão affirment que ces études ont démontré, du point de vue structurel, (1) que les variantes [i] et [u] - dont la productivité dans ces localités varient entre $10 \%$ et $24 \%$-, aussi bien que les variantes $[\varepsilon]$ et [0] sont motivées par l'harmonisation vocalique avec une voyelle, respectivement, haute ou basse dans la syllabe ultérieure, et (2) que la réduction vocalique, en plus des restrictions d'ordre lexical, obéit à des contraintes liées au point et au mode d'articulation des consonnes adjacentes. 


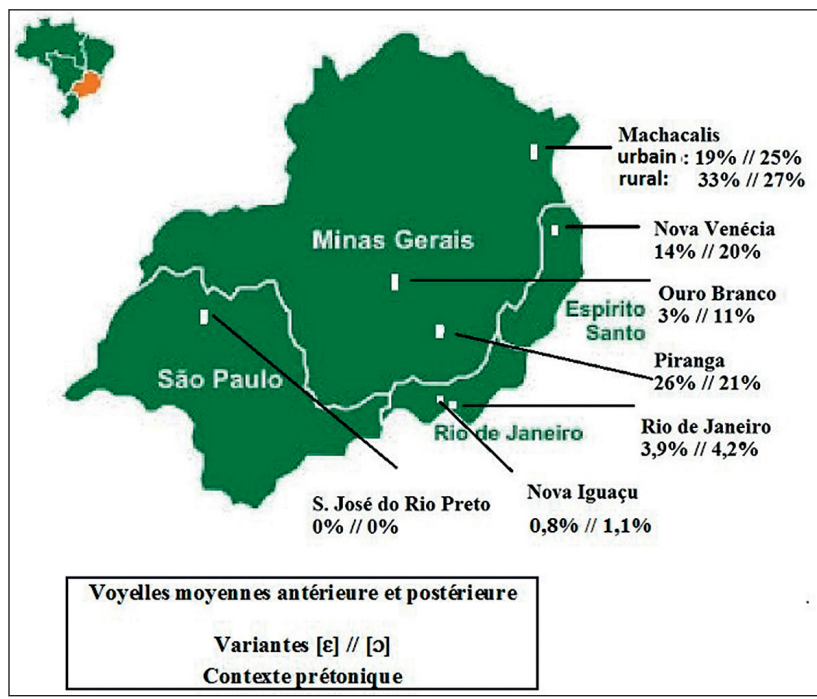

Figure 3. - Fréquence des variantes [ $\varepsilon$ ग] prétoniques dans les parlers de la région Sud-Est.

Source : Rocha et Brandão (2014, inédit), ayant comme base la carte sur $<$ www.brasilescola.com/brasil> (avec des changements).

Du point de vue diatopique, on observe des aspects divergents indiquant une plus grande complexité de Minas Gerais et probablement de l'Espírito Santo face à Rio de Janeiro/Nova Iguaçu et São Paulo. Ceci suggère l'existence de différentes aires linguistiques, en particulier en ce qui concerne le premier des États mentionnés. L'élément de différenciation par rapport aux parlers de ces localités est la fréquence des variantes moyennes ouvertes. Comme le montre la figure 3, dans deux localités de Minas Gerais (Machacalis et Piranga), [ $\varepsilon$ o ] atteignent des taux entre $19 \%$ et 33\%, et dans l'Espírito Santo (Nova Venécia), entre $14 \%$ et $20 \%$. Dans la région métropolitaine de Belo Horizonte (Ouro Branco), les taux sont compris entre $3 \%$ et $11 \%$, et il y a une chute drastique à Rio de Janeiro (dans le parler cultivé et à Nova Iguaçu, respectivement $3,9 \%$ et $0,8 \%$ pour [ $[\varepsilon]$ et $4,2 \%$ et $1,1 \%$ pour [o]), limitée à certains éléments lexicaux. Enfin, à São Paulo (São José do Rio Preto), ces variantes ne sont pas enregistrées.

En ce qui concerne ce cadre, ce qui est enregistré à Machacalis et Nova Venécia n'est pas si surprenant, car ces villes sont à proximité de Bahia où les variantes $[\varepsilon$ o ] prédominent largement. On ne peut pas en dire autant de Piranga, située à proximité des zones où [e o] sont dominantes. Toutefois les taux de $[\varepsilon$ o $]$ relevés à Rio de Janeiro et Nova Iguaçu sont observés dans un nombre limité d'éléments lexicaux qui présentent une voyelle tonique ouverte, comme $\mathrm{b}[0]^{\prime} 1[0] \mathrm{ta}, \mathrm{P}[\varepsilon]^{1} 1[\varepsilon]$, entre autres. 
La variation des voyelles moyennes qui, dans tous les parlers brésiliens, peut être décrite comme stable, contraste avec le cas des rotiques. Ce dernier, très complexe aussi, comprend deux processus : la fricatisation et l'effacement, des différentes étapes de changement en cours en fonction du dialecte et de la position contextuelle du segment.

Callou (1987) a démontré que, dans le parler cultivé de la ville de Rio de Janeiro dans les années 1970, la fricative vélaire était prédominante, sauf en position de coda externe (comme dans falar), dans laquelle la fricative glottale et l'effacement étaient les variantes plus fréquentes. Parmi ses conclusions, elle déclare que le changement de vibrante à fricative était plus avancé chez les femmes dans tous les contextes, en particulier dans l'attaque initiale de mot (comme dans roda). En outre, la banlieue de la ville et les sujets plus jeunes étaient d'autres facteurs qui étaient en train de conditionner le processus en cours de postériorisation. Plus tard, Callou, Leite et Moraes (2002) ont analysé les données des années 1990 faisant référence non seulement à Rio de Janeiro, mais aussi à quatre autres capitales, concluant que, dans quelques parlers, la fricative glottale avait déjà dépassé la variante vélaire, y compris dans l'attaque syllabique. En ce qui concerne le rotique dans la coda interne (comme dans carne), ils ont constaté que le processus en cours de postériorisation permettait d'opposer, au moins en termes de pourcentages, deux zones (voir fig. 4) : une, plus au sud, à laquelle appartiennent les villes de Porto Alegre (POA) et São Paulo (SP), et l'autre plus au nord où se trouvent les trois autres villes de Rio de Janeiro (RJ), Salvador (SSA) et Recife (RE).

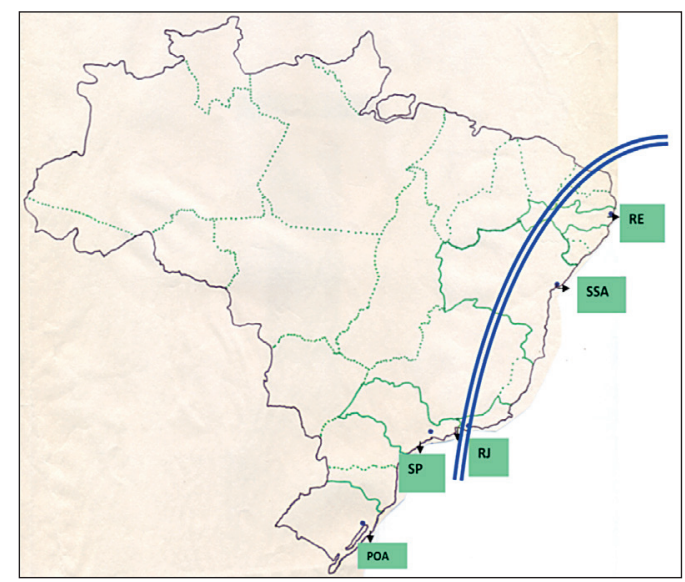

$\mathrm{POA}=$ Porto Alegre $; \mathrm{SP}=$ São Paulo $; \mathrm{RJ}=$ Rio de Janeiro $; \mathrm{SSA}=$ Salvador $; \mathrm{RE}=$ Recife .

Fig. 4. - Tendance moins forte (POA et SP) ou plus forte (RJ, SSA et RE) à la postériorisation du rotique dans la coda interne dans les parlers de 5 capitales brésiliennes.

Source : Callou \& Brandão (2013, inédit), adapté de Callou \& Moraes (1995; p. 73). 
Dans le tableau $1^{3}$ ci-dessous, la gamme de variation des rotiques est plus explicite selon le contexte dans les parlers de ces cinq capitales. On observe que les fricatives dominent dans toutes les positions, même si les vibrantes montrent encore des contextes de résistance à São Paulo et Porto Alegre, respectivement, l'uvulaire dans le contexte prévocalique et l'alvéolaire dans le contexte intervocalique et prévocalique. Dans le contexte postvocalique à la fin des mots, où prédomine l'effacement, dans ces deux villes, la consonne battue alvéolaire persiste encore.

\begin{tabular}{|c|c|c|c|c|c|c|}
\hline Contexte & Exemple & Rio de Janeiro & São Paulo & Porto Alegre & Salvador & Recife \\
\hline Intervocalique & carro & $\begin{array}{c}\text { fric. vélaire } \\
\text { (fric. glottale) }\end{array}$ & fric. vélaire & $\begin{array}{l}\text { fric. vélaire } \\
\text { (vibr. } \\
\text { alvéolaire) }\end{array}$ & $\begin{array}{l}\text { fric. glottale } \\
\text { (fric. vélaire) }\end{array}$ & $\begin{array}{l}\text { fric. glottale } \\
\text { (fric. vélaire) }\end{array}$ \\
\hline $\begin{array}{c}\text { Pré-vocalique } \\
\text { initial / milieu } \\
\text { de mot }\end{array}$ & $\begin{array}{l}\text { roda } \\
\text { Israel }\end{array}$ & $\begin{array}{l}\text { fric. glottale } \\
\text { (fric. vélaire) }\end{array}$ & $\begin{array}{l}\text { fric. vélaire } \\
\text { (vibr. } \\
\text { uvulaire) }\end{array}$ & $\begin{array}{l}\text { fric. vélaire } \\
\text { (vibr. } \\
\text { alvéolaire) }\end{array}$ & fric. glottale & fric. glottale \\
\hline $\begin{array}{c}\text { Post-vocalique/ } \\
\text { médial de mot }\end{array}$ & $\begin{array}{l}\text { carta } \\
\text { carne }\end{array}$ & $\begin{array}{c}\text { fric. vélaire } \\
\text { (fric. glottale) }\end{array}$ & $\begin{array}{l}\text { battue } \\
\text { alvéolaire }\end{array}$ & $\begin{array}{c}\text { battue } \\
\text { alvéolaire }\end{array}$ & $\begin{array}{l}\text { fric. glottale } \\
\text { (fric. vélaire) }\end{array}$ & $\begin{array}{l}\text { fric. glottale } \\
\text { (fric. vélaire) }\end{array}$ \\
\hline $\begin{array}{l}\text { Post-vocalique } \\
\text { final de mot }\end{array}$ & $\begin{array}{c}\text { cantor famoso } \\
\text { cantor novo } \\
\text { bom cantor }\end{array}$ & {$[\varnothing]$} & $\begin{array}{c}{[\varnothing]} \\
\text { (battue } \\
\text { alvéolaire) }\end{array}$ & $\begin{array}{c}\text { battue } \\
\text { alvéolaire }\end{array}$ & {$[\varnothing]$} & [Ø] \\
\hline \multicolumn{7}{|c|}{ (Sans tenir compte de contraintes structurelles/extralinguistiques) } \\
\hline
\end{tabular}

Tableau 1. - Réalisations plus fréquentes de $R$ selon le contexte dans les parlers de cinq capitales brésiliennes.

Selon Callou, «o apagamento que se observa em contexto de coda silábica externa constitui o estágio final de um processo de enfraquecimento que leva à simplificação da estrutura silábica no PB: $R \rightarrow h \rightarrow \emptyset e$, consequentemente, $C V C \rightarrow C V^{4} \gg(2015$, sous presse). Ce processus est à un stade plus avancé dans les verbes à l'infinitif.

Bien que présentés ici d'une façon assez résumée, les résultats concernant ces deux variables qui peuvent contribuer, d'une certaine façon, à délimiter des aires linguistiques, illustrent, d'autre part, la nécessité d'envisager, en plus de raisons structurelles, des aspects d'ordre social pour une meilleure appréhension des facteurs qui motivent les variantes. Dans ce sens, la sociolinguistique variationnelle est d'une grande valeur.

3. On a pris en compte des taux de pourcentage obtenus par Callou et Moraes (1995). Lorsqu'une variante est entre parenthèses, cela indique que la différence entre elle et celle indiquée comme la plus fréquente est, au maximum, de $2 \%$.

4. «[...] l'effacement observé dans le contexte de coda syllabique externe est l'étape finale d'un processus d'affaiblissement qui mène à la simplification de la structure syllabique dans le $\mathrm{PB}: \mathrm{R} \rightarrow \mathrm{h} \rightarrow \varnothing$ et, en conséquence, $\mathrm{CVC} \rightarrow \mathrm{CV}$.» 


\section{2. À propos de quelques aspects de la société brésilienne actuelle}

Un demi-siècle après les premières études de base sociolinguistiques, qui, de manière pionnière, cherchaient à combiner les composantes géo et sociolinguistique, il est nécessaire de présenter, même brièvement, quelquesunes des principales caractéristiques de la société brésilienne actuelle afin de mieux évaluer les problèmes rencontrés par ceux qui se consacrent aux études de variation dans le pays.

Le Brésil, qui fut pendant quatre siècles un vaste pays rural selon les mots de Celso Cunha (1972; p. 58), a maintenant sa population concentrée dans des centres urbains, cadre défini à partir des années 1960, dans le SudEst, et seulement à partir des années 1980 dans les autres régions. Le processus a été abrupt dans certaines régions, comme dans le Centre-Ouest et le Sud, et progressif dans le Nord, le Nord-Est et le Sud-Est. Le graphique qui compose la figure 6 (basé sur le tableau fourni par l'IBGE ${ }^{5}$ ) montre cette transformation radicale dans un court laps de temps.

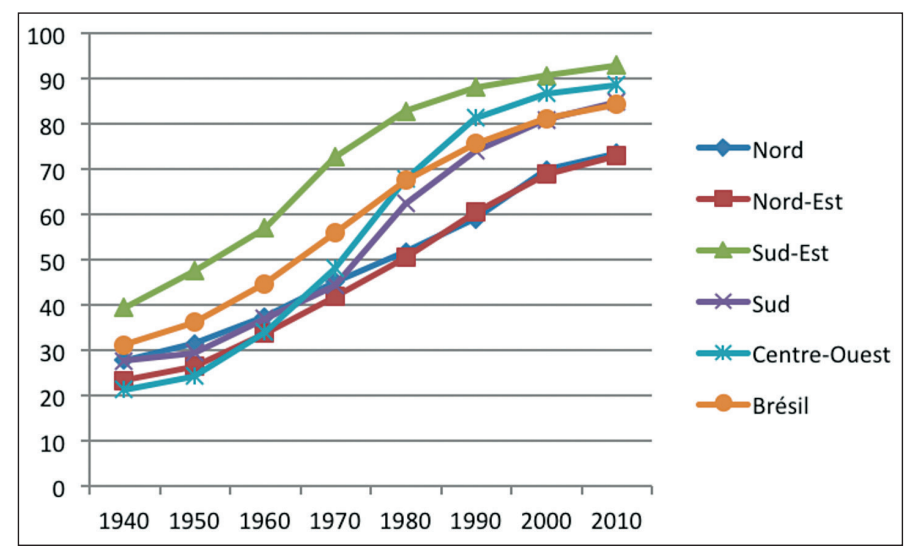

Figure 5. - Brésil : taux d'urbanisation par régions en pourcentage.

Basé sur un tableau fourni par l'IBGE

(<http://seriesestatisticas.ibge.gov.br/series.aspx?vcodigo=POP122\&t=taxa-urbanizacao $>$ ).

Outre la mobilité de la population des zones rurales vers les zones urbaines dans les différentes régions, il faut aussi considérer les déplacements interrégionaux découlant de la recherche de nouvelles opportunités d'emploi. Dans la région Sud-Est, par exemple, se trouvent les deux plus

5. Instituto Brasileiro de Geografia e Estatística (voir <www.ibge.gov.br>). 
grandes régions métropolitaines du pays : São Paulo, avec 20935204 habitants et Rio de Janeiro, avec 11973505 , où la concentration de la population urbaine atteint le taux le plus élevé : 99,3\%. La grande partie de ce contingent provient d'autres régions du pays, en particulier du Nord-Est et de Minas Gerais, ce qui rend ces espaces extrêmement complexes non seulement d'un point de vue social, mais aussi linguistique, puisque il y a un contact multidialectal intense dans ces aires.

$\mathrm{Au}$ cours de la dernière décennie, en raison de politiques inclusives mises en pratique par le gouvernement et qui ont abouti à un plus grand pouvoir d'achat pour la population à faible revenu, a émergé ce qu'on est en train d'appeler la nouvelle classe moyenne - pour certains, nouvelle classe ouvrière - dont les caractéristiques ne sont pas encore bien définies, bien que quelques études aient essayé de la délimiter (Porchmann, 2012; Yaccoub, 2011; Neri, 2010, parmi d'autres). Bien que ce changement social important ait abouti à un meilleur accès aux biens de consommation et à une meilleure qualité de vie, un tel segment, comme indiqué par certains sociologues (Neri, 2010), a des valeurs - en particulier en ce qui concerne les traits de comportement, les biens culturels et les convictions politiques - bien différentes de celles de la classe moyenne traditionnelle, généralement constituée de professionnels libéraux. En ce sens, le niveau d'éducation s'avère être la variable sociale la plus appropriée pour l'observation de la réalité linguistique du Brésil, qui est hétérogène et polarisée (Lucchesi, 2003) : sur cette base, on peut opposer, grosso modo, des variétés sociolinguistiquement divergentes, comme populaires/élevées, urbaines/rurales. Cette dernière dichotomie, apparemment diatopique, en réalité est sociale par essence, puisque les zones dites rurales sont constituées, en règle générale, par des individus qui, outre de ne pas vivre dans des communautés de grande stratification sociale, ont peu ou pas d'accès à l'éducation formelle.

Dans la figure 7, sur la base de données de l'IBGE, sont exposés les pourcentages concernant la distribution de la population par années d'études au Brésil et dans les régions métropolitaines de Rio de Janeiro (M.R. RJ) et São Paulo (M.R. SP). On constate que, dans ces deux derniers domaines, il n'y a pas de différences significatives, les taux de pourcentage étant très similaires. Les différences sont plus claires lorsque l'on compare les deux régions à ce qu'on observe au Brésil en général : les indices concernant les pôles du continuum de l'éducation montrent plus de distance entre les individus plus ou moins instruits et, encore, l'existence d'un nombre considérable d'individus qui n'ont pas fini le premier cycle de l'enseignement fondamental (quatre années) ou le deuxième (quatre années de plus). D'autre part, seulement $10,10 \%$ de la population ont des diplômes universitaires. 


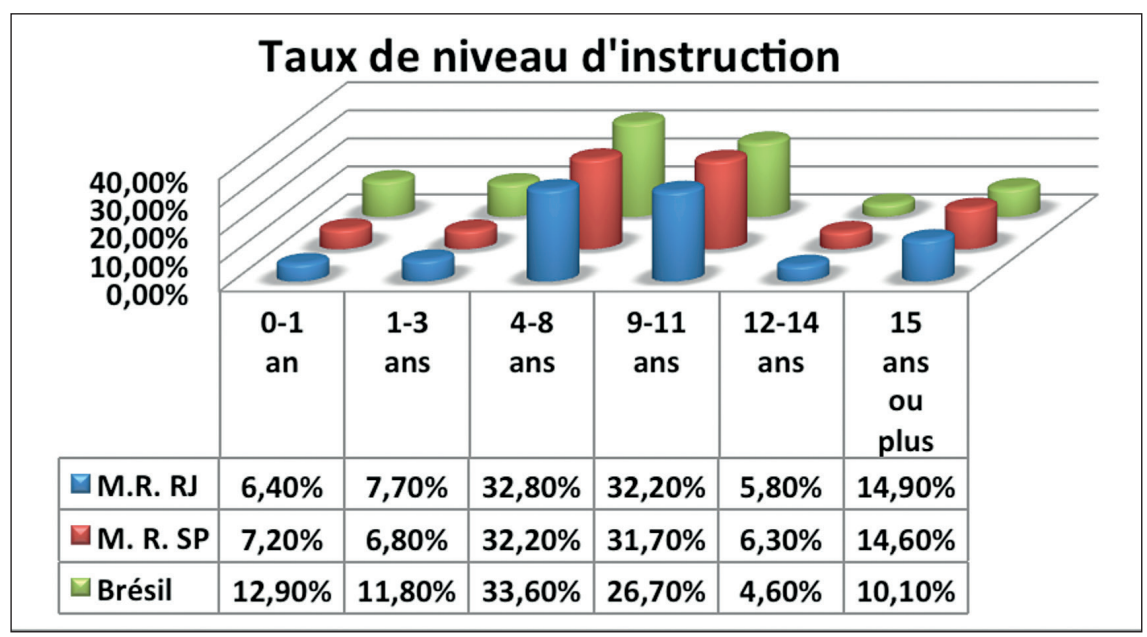

M.R. RJ = Région métropolitaine de Rio de Janeiro.

M.R. SP = Région métropolitaine de São Paulo.

Figure 6. - Brésil : taux de niveau d'instruction.

Source : IBGE.

Trois facteurs, combinés aux caractéristiques déjà signalées dans la formation du PB, ont contribué à la grande complexité linguistique que l'on observe aujourd'hui au Brésil : la grande mobilité spatiale qui a concentré la population dans les zones urbaines, la mobilité sociale qui a plus récemment déterminé l'émergence d'une nouvelle classe, ainsi que l'accès accru à l'éducation formelle à tous les niveaux en particulier dans les années 1980.

Bien que le PB soit caractérisé, comme une variété hétérogène et polarisée, les différences entre les variétés populaires et les variétés cultivées ne sont pas toujours nettes: on observe des intersections, parfois subtiles, entre ces deux pôles. Bortoni-Ricardo (2004, p. 51-70) affirme que, pour une meilleure compréhension de la nature multidimensionnelle des phénomènes variables au Brésil, on devrait envisager trois continua : le continuum d'urbanisation (variétés rurales $\rightarrow$ zones urbaines $\rightarrow$ variétés urbaines standard); le continuum d'oralité-littératie (événements d'oralité $\rightarrow$ événements de littératie) et le continuum de contrôle stylistique (moins contrôlé $\rightarrow$ plus contrôlé). À ceux-là, on pourrait ajouter (Brandão, 2013) un continuum de niveau d'instruction, qui peut se résumer en deux pôles (zéro/bas $\rightarrow$ haut), avec deux segments intermédiaires, correspondant à 5-8 années et 9-11 années de scolarité. 


\section{3. À propos de la pertinence d'une perspective géo-sociolinguistique}

Bien qu'encadré dans une perspective traditionnelle, puisque l'Atlas Prévio dos Falares Baianos - APFB (Rossi et al., 1963) ne favorisait que la dimension diatopique et que les données enregistrées étaient fournies par des informateurs du type NORM $^{6}$ (Chambers \& Trudgill, 1980, p. 33), on peut affirmer que cet atlas est la première étude vraiment rigoureuse d'un parler brésilien, car elle a été effectuée d'après des paramètres bien définis. L'Atlas Lingüístico de Sergipe - ALSE (Ferreira et al., 1987), volume 1, développé par la même équipe, dans les années 1960, mais publié seulement en 1987, a déjà pris en compte la variable sexe dans le choix des informateurs, ce qui peut être considéré comme une première étape vers l'élargissement de la représentativité sociale des données recueillies.

Toutefois, pendant la période entre ces deux initiatives et le séminaire «Chemins et perspectives pour la géolinguistique au Brésil», durant lequel en 1996, a été officialisée l'idée d'élaborer l'Atlas linguistique du Brésil - rares étaient les projets développés dans le cadre de la géographie linguistique - qui, en quelque sorte, était devenu l'arrière-plan en face du développement remarquable dans le pays, de la recherche sociolinguistique d'inspiration labovienne.

Les quelques atlas construits ou en préparation à cette étape-là et qui plus tard ont été publiés - sauf pour l'Atlas linguistique de Ceará-ALECE (Bessa, 2010), ayant pour but d'observer le changement en tenant compte non seulement du sexe, mais aussi du niveau d'instruction (analphabètes/ alphabétisés) et de l'âge (30/60 ans) - sont restés dans une perspective essentiellement diatopique : l'Atlas Lingüístico-Etnográfico da Região Sul (Koch et al., 2002), l'Esboço de um Atlas Lingüístico de Minas Gerais (Ribeiro et al., 1977), l'Atlas Lingüístico da Paraíba (Aragão \& Menezes, 1984), ont suivi l'exemple de l'ALSE, comme cela a été le cas du premier volume de l'Atlas Lingüístico do Paraná (Aguilera, 1994).

Cependant, à la fin des années 1980, un projet se voulait différent des autres, cherchant à caractériser les restrictions de nature diverse qui soustendent la mise en œuvre des variantes : le projet APERJ (Atlas Etnolingüístico dos Pescadores do Estado do Rio de Janeiro $)^{7}$ qui n'a jamais été

6. Sédentaire (nonmobil), plus vieux (older), rural (rural) et du sexe masculin (homme) et, en général, illettré.

7. Ce projet a été conçu par une équipe de cinq professeurs de l'Universidade Federal do Rio de Janeiro. 
publié. Il a été établi un réseau de 45 points d'enquête, composés chacun de six informateurs masculins répartis selon trois groupes d'âge (18-35, 36-55 et 56 ans et plus). Le questionnaire comportait 422 questions qui comprenaient, essentiellement, le lexique lié à l'activité de pêche distribué en trois grands champs sémantiques - l'environnement physique, l'environnement biotique (flore et faune) et l'environnement anthropique (l'homme et l'activité). On s'attendait également à obtenir des énoncés libres d'environ 15 à 20 minutes.

On cherchait une approche tridimensionnelle qui permettrait non seulement de voir les variantes dans leur distribution horizontale (dans l'espace géographique) et temporelle (par la performance des individus de différents groupes d'âge), mais aussi de les observer en profondeur, car, sur la base des réponses au questionnaire et des élocutions libres, seraient prises en compte les occurrences dans le corpus des variantes de quelques variables linguistiques (comme /R/ et /S/ en coda syllabique) et, par conséquent, leurs facteurs conditionneurs seraient indiqués. Cette dernière décision impliquait d'élaborer des analyses ponctuelles selon la méthode variationnelle. L'idée était d'organiser, en plus de cartes lexicales, deux types de cartes phonétiques : analytiques, avec les réponses au questionnaire, et synthétiques, avec des résultats concernant les variables linguistiques plus importantes, par exemple /R/ et /S/ en coda syllabique.

Initié dans une phase où, comme on l'a dit, les projets dans la ligne de la géolinguistique étaient rares au Brésil, l'équipe a osé proposer de combiner la méthodologie de la sociolinguistique variationnelle et celle de la géolinguistique. Dans des travaux présentés à quelques congrès, avec des ressources manuelles, parce qu'il n'y avait pas d'applications informatiques comme celles d'aujourd'hui, des cartes linguistiques ont été organisées d'après des taux de pourcentage et poids relatifs découlant de l'utilisation, à ce moment-là, du programme Varbrul. On peut dire que ces cartes, complémentées par l'analyse des facteurs qui contribuent à la variation, avaient déjà un caractère interprétatif.

Brandão (1994) a présenté un total de sept cartes synthétiques sur les rotiques dans le parler de treize localités des régions Nord et Nord-est de l'État. L'une d'entre elles, portant le $\mathrm{n}^{\circ} 2$, concernant la consonne battue alvéolaire en contexte post-vocalique interne, sert d'exemple à la conception des cartes (voir fig. 7 a-b). En (b) apparaît la distribution des données basée sur les pourcentages par groupe d'âge (chaque colonne correspond à un groupe d'âge et chaque carré, vertical, correspondant à $20 \%$ ); en (a) sont indiqués, d'une façon synthétique, les indices relatifs à l'analyse variationnelle de la variante mise au point. 
(a) Indicateurs

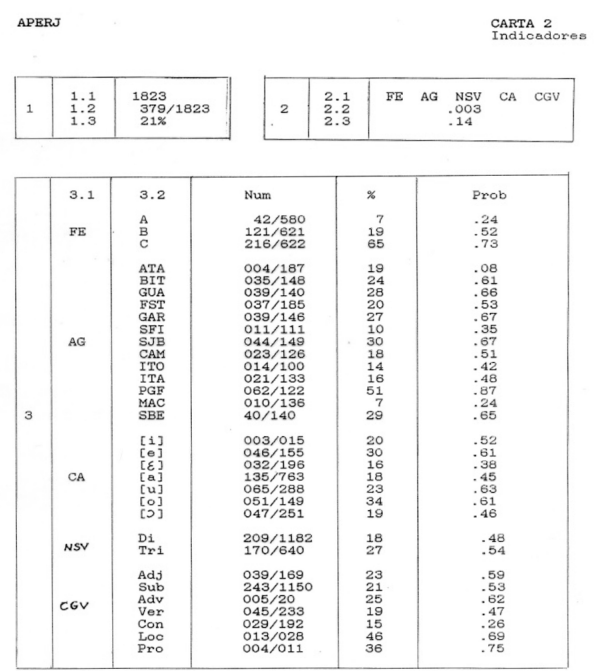

(b) Visualisation des taux de pourcentage

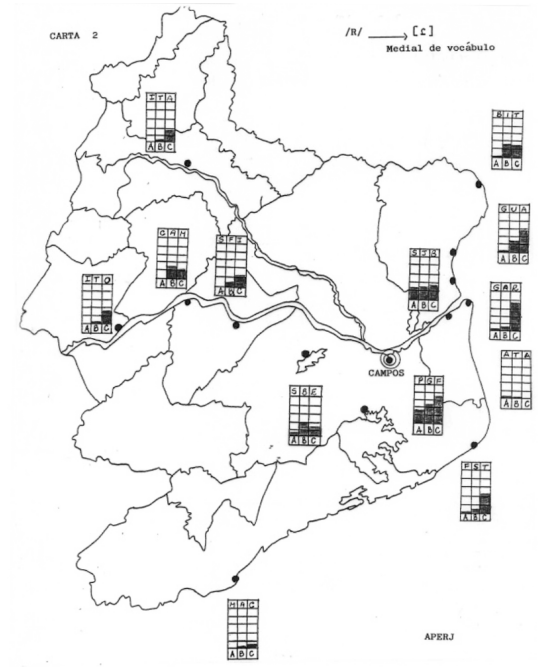

Figure 7 ( $a$ et b). - Carte $n^{\circ} 2$ de l'APERJ :/R/ réalisé comme consonne battue alvéolaire, en contexte post-vocalique interne.

À l'arrière de la carte 1, apparaît ce qu'on a appelé les indicateurs de la carte 2 : au champ 1, le nombre total d'occurrences de R (1.1) en coda interne, (1.2) de la consonne battue alvéolaire dans ce contexte et le pourcentage qui lui correspond. Au champ 2, les variables pertinentes pour l'application de la consonne battue alvéolaire (2.1): FE (groupe d'âge), AG (aire géographique), NSV (nombre de syllabes du mot), CA (contexte antécédent) et AGB (classe grammaticale du mot); (2.2) la signifiance de l'analyse quantitative, et (2.3) l'input de la règle. Au champ 3, sont repris et ventilés, également au moyen de sigles, les variables sélectionnées et leurs facteurs respectifs, en indiquant le nombre, le pourcentage et le poids relatif/probabilité (Prob) correspondant à chacun d'eux. Chaque carte a été l'objet de commentaires qui expliquaient les données synthétiquement indiquées.

La méthodologie proposée pour la préparation de l'APERJ, exposée ci-dessus, qui s'éloigne des tendances de l'époque, pouvait être considérée à ce moment-là, dans une certaine mesure, hérétique ou bizarre. Bien sûr, un tel projet, en fonction de la zone à couvrir, est coûteux non seulement en termes financiers mais aussi en ce qui concerne le temps d'exécution. Cependant, aujourd'hui, face au développement et aux contributions de la linguistique, on voit que des études variationnelles doivent nécessairement considérer au moins les variables sociales qui sont les plus importantes dans une zone géographique donnée. 
Bien que les cartes de l'atlas n'aient pas été publiées, le corpus obtenu a formé la base de plusieurs études de nature géo-sociolinguistique développées comme mémoires et/ou thèses, ou publiées sous la forme de chapitres de livres et/ou d'articles de revues. Les parlers du Nord et du Nord-Ouest de l'État de Rio de Janeiro, représentés par 13 communautés, ont été décrits au moyen de ces recherches, par rapport aux variables phonologiques (/R/ et $/ \mathrm{S} /$ en coda, les occlusives dentales, la latérale antérieure et la postérieure, entre autres) et morphosyntaxiques. Un résumé des principales caractéristiques des parlers de ces régions se trouve chez Brandão (2008).

Tel que mentionné par Callou, l'idée que la dialectologie et la sociolinguistique sont «deux perspectives d'observation de la langue qui ne s'opposent pas, mais se rencontrent et se complètent» commence à devenir explicite à la fin des années 1960 :

O desenvolvimento do famoso Projeto da norma linguística urbana culta (Projeto NURC), que teve início no final da década de 60 e início da de 70, é prova cabal desse fato. Este Projeto surge a partir da nova orientação dos dialectólogos para os países do chamado Novo Mundo e da concepção de uma Dialetologia urbana elou Sociolinguística, sob a inspiração do Proyecto de Estúdio Coordinado de la Norma Linguística Culta de las Principales Ciudades de Iberoamérica y de la Península Ibérica. Em relatório solicitado pela Comissão de Linguística Iberoamericana do PILEI, em 1968, o Professor Nélson Rossi, da UFBA, afirmava a conveniência em se estender o projeto ao Brasil, em virtude de serem tão evidentes e tão relevantes os pontos comuns à problemática do espanhol nas Américas e do Português do Brasil ${ }^{8}$. (2010, p. 35)

D'une certaine façon, toujours d'après Callou (2010, p. 34), le lien entre les approches dialectologique et sociolinguistique est né de l'intérêt à décrire le parler urbain, et donc, du besoin de créer une nouvelle méthodologie pour l'étude des langues romanes, tel que revendiqué par Rona et Diego Catalán.

8. «Le développement du fameux projet de la norme linguistique urbaine cultivée (projet NURC), qui a commencé à la fin des années 1960 et au début des années 1970, est une preuve suffisante de ce fait. Ce projet résulte de la nouvelle conception des dialectologues pour les pays du dit Nouveau Monde et de la conception d'une dialectologie urbaine et/ou sociolinguistique, sous l'inspiration du Proyecto de Estúdio Coordinado de la Norma Linguística Culta de las Principales Ciudades de Iberoamérica y de la Península Ibérica. Dans un rapport demandé par le Comité de linguistique ibéro-américaine du PiLEI, en 1968, le professeur Nélson Rossi, de l'UFBA, affirmait la convenance d'étendre le projet au Brésil, en raison des évidents et pertinents points communs de la problématique de l'espagnol dans les Amériques et de celle du portugais au Brésil.» 
La discussion sur ce que Radtke et Thun ont appelé «une crise dans la géolinguistique romane contemporaine» (1996) les a amenés à réaliser en 1991, le symposium «Les nouveaux chemins de la géolinguistique romane», pour faire un bilan de ce qui avait été fait jusqu'à ce momentlà dans ce domaine, et de rendre possible «la réunion des représentants de la vieille géographie linguistique monodimensionnelle avec les novateurs» (1996, p. 27). À la suite de ces constatations, chaque jour plus évidentes, qu'il fallait représenter, directement, les divers types de motivations (diagénériques, diastratiques, diaphasiques, etc.) qui régissent l'occurrence de formes variantes et, en particulier de la sociolinguistique, il a été proposé que la géolinguistique se rénove en adoptant explicitement une perspective pluridimensionnelle (Radke \& Thun, 1996), mieux adaptée à la réalité que l'on observe dans les Amériques. Dans ce symposium, plusieurs dialectologues, comme Moreno Fernandez, Harald Thun, Lope Blanch ont rapporté de nouvelles expériences par rapport à la méthodologie de la géolinguistique, toujours plus proche des concepts sociolinguistiques.

Mise en œuvre dans l'ADDU - Atlas Lingüístico Diatópico y Diastrático del Uruguay - , la nouvelle méthodologie pluridimensionnelle a eu une grande répercussion au Brésil, où, depuis lors, les atlas linguistiques (y compris l'Atlas Lingüístico do Brasil) n'ont pas manqué d'utiliser des variables sociales. Comme le montre Elizaicín :

Hoy, con la unión casi íntima de la geolingüística y la sociolingüística (a lo que hay que añadir la lingüística histórica) el ordenado esquema anterior, y los limites y alcances de cada disciplina (o actividad científica) empiezan a desdibujarse. Ello es consecuencia del frenético desarrollo de muchas ramas de la lingüística en los últimos decenios del siglo pasado, las que privilegian en forma notoria la visión de la lengua en uso, en la cotidianeidad, en el âmbito más cercano del diálogo y la interacción, etc. ${ }^{9}$. (2010, p. 18)

Tout en reconnaissant une certaine inégalité épistémologique entre la géolinguistique et la sociolinguistique, Elizaicín déclare qu'elles peuvent converger,

9. «Aujourd'hui, avec l'union presque intime de la géolinguistique et de la sociolinguistique (à laquelle il convient d'ajouter la linguistique historique) le schéma ordonné précédent, et les limites et la portée de chaque discipline (ou activité scientifique) commencent à s'atténuer. Cela est dû au développement frénétique de nombreuses branches de la linguistique : dans les dernières décennies du siècle dernier, celles qui privilégient nettement la vision de la langue utilisée dans la vie quotidienne dans le milieu le plus proche du dialogue et de l'interaction, etc.» 
[...] ya que la concepción general del lenguaje que ambas sustentan es, en el fondo, la misma, a saber, el lenguaje es un objeto móvil por definición, altamente sensible a la acción e influencia de factores externos que imponen modificaciones lingüísticas de mayor o menor relevância o aceptación por parte de la comunidad usuaria de ese linguaje ${ }^{10}$. (2010, p. 18-19)

Au Brésil, même si l'habitude d'intégrer des variables sociales à la méthodologie des atlas linguistiques a pris du temps, dans le cadre des premières études de nature sociolinguistique, la variable diatopique était parfois présente, comme dans l'étude pionnière sur le /S/ en coda syllabique dans le parler de Rio de Janeiro, réalisée par Callou et Marques (1975), et où sont pris en considération, comme une variable, six quartiers de la ville. L'étude a même montré que, dans Madureira et la zone Sud, où l'interaction socioculturelle et spatiale est plus prononcée (p. 133), chez les femmes, il y a une plus grande oscillation entre la variante palatalisée et les non palatalisées. Comme le remarque Brandão :

La totalité de la connaissance sur la dynamique des parlers réunie depuis qu'ont été définies, à la fin du XIX ${ }^{\mathrm{e}}$ siècle, les bases de la géographie linguistique est une monnaie à double face. Si, d'une part, elle fournit au chercheur un ensemble précieux de paramètres qui lui permettent de faire des choix méthodologiques appropriés à la zone où il veut enregistrer/interpréter la variation linguistique, elle intervient, de l'autre, dans leur choix, étant donné la complexité de son objet d'étude.

Si l'on ajoute les apports de la Dialectologie à ceux du champ de la Sociolinguistique, on doit admettre que des options méthodologiques impliquent toujours la conscience des limitations imposées par une telle complexité, mais sans renoncer pour autant à y appliquer de nouvelles approches analytiques, pour essayer de comprendre des questions qui ne seront peut-être dûment évaluées et approfondies que dans l'avenir. (2013,p. 5)

L'intime relation entre la sociolinguistique et la géolinguistique est indéniable et peut servir de fondement, à double sens, aux chercheurs qui développent prioritairement des études soit dans l'un ou l'autre champ d'étude. Labov, par exemple, qui a cherché dans le LANE les premières informations pour son étude sur Martha's Vineyard, a publié en 2006, avec Sharon

10. «[...] puisque la conception générale de langage que les deux soutiennent est essentiellement la même, à savoir, le langage est un objet mobile par définition, très sensible à l'action et l'influence des facteurs externes qui imposent des changements linguistiques plus ou moins pertinents ou l'acceptation par la communauté d'usagers de ce langage.» 
Ash et Charles Boberg, l'Atlas de l'anglais nord-américain : phonétique. Phonologie et changement sonore (ANAE) ${ }^{11}$, bénéficiant des nombreuses études qu'il a effectuées sur l'anglais américain.

\section{Conclusion}

L'étude de la variation a toujours impliqué la recherche des motivations des formes variantes. Gilliéron non seulement les a enregistrées sur les cartes, mais a aussi développé une série d'études pour les interpréter, en s'appuyant sur la dimension diatopique. D'autre part, à la même époque, l'étude sur le parler de Charmey, une commune suisse, publiée en 1905 par Gauchat, considérée comme un travail précurseur des idées sociolinguistiques, a déjà démontré que la variation pourrait être attribuée à des facteurs liés aux variables sexe, profession, tranche d'âge et position sociale.

Non seulement la distance spatiale, comme Trudgill l'a noté, peut, par exemple, déterminer la diffusion de traits linguistiques :

Distance, then is clearly an important factor in the spread of linguistic forms, although in many cases social distance may be as important as geographical distance [...]: two towns may be socially "closer" to each other then they are to the intervening stretches of the countryside ${ }^{12} .(1987, \mathrm{p} .172)$

Aujourd'hui, où l'on peut compter non seulement sur plusieurs informations concernant la structure et la dynamique des langues, mais aussi sur des ressources informatiques chaque jour plus fonctionnelles et puissantes, essayer de définir, de la façon la plus vaste possible, les différents facteurs (sociaux, spatiaux et structurels) qui contribuent à l'occurrence de variantes dans une communauté linguistique donnée est un impératif qui ne peut échapper au linguiste cherchant à la comprendre et à détecter de possibles processus de changement. En ce sens, une approche géo-sociolinguistique, non seulement pour le cas brésilien, semble être la plus appropriée pour l'étude de la variation, en particulier dans le monde actuel, où des changements technologiques et comportementaux imposent, dans un laps de temps très court, des nouvelles dynamiques aux groupes sociaux et, même, de nouvelles configurations spatiales.

11. Voir la page internet de Labov (<www.ling.upenn.edu/ labov>) et le site de Mouton de Gruyter, qui a publié l'atlas.

12. «Distance, donc, est clairement un facteur important dans la propagation des formes linguistiques, bien que dans de nombreux cas la distance sociale peut être aussi importante que la distance géographique $[\ldots]$ : deux villes peuvent être socialement «plus proches» l'une de l'autre qu'elles le sont de leurs environs.» 


\section{RÉFÉRENCES BIBLIOGRAPHIQUES}

Aguilera Vanderci de Andrade, 1994, Atlas Lingüístico do Paraná, vol. 1, Curitiba, Imprensa Oficial do Paraná.

Almeida Luciana de Fátima, 2008, A variação das vogais médias pretônicas na cidade mineira de Machacalis, mémoire de master, Universidade Federal de Minas Gerais, Belo Horizonte.

Alves Marlúcia Maria, 2008, As vogais médias em posição pretônica nos nomes no dialeto de Belo Horizonte: estudo da variação à luz da Teoria da Otimalidade, thèse de doctorat, Universidade Federal de Minas Gerais, Belo Horizonte.

Amaral Amadeu, 1976 [1920], O dialeto caipira, São Paulo, Hucitec, Secretaria de Cultura, Ciência e Tecnologia, $3^{\mathrm{e}}$ éd.

Aragão Maria do Socorro Silva \& Menezes Cleusa P. B, 1984, Atlas Lingüístico da Paraíba, vol. 1 : Cartas léxicas e fonéticas et vol. 2 : Análise das formas e estruturas linguísticas encontradas, Brasilia, CNPq/UFPB.

Bessa José Rogério Fontenele, 2010, Atlas Lingüístico do Estado do Ceará, Fortaleza, UFC, 2 vol.

Bortoni-Ricardo Stella Maris, 2004, Educação em língua materna: a sociolinguística na sala de aula, São Paulo, Parábola Editorial.

BRANDÃo Silvia Figueiredo, 1994, «Algumas cartas fonéticas do Atlas Etnolingüístico dos Pescadores do Estado do Rio de Janeiro », Congresso Internacional sobre o Português, Associação Portuguesa de Linguística, Universidade de Lisboa.

BRANDÃo Silvia Figueiredo, 2013, «Réalité sociolinguistique brésilienne et géolinguistique pluridimensionnelle», dans E. Carrilho, C. Magro et X. Álvarez (éds), Current Approaches to Limits and Areas in Dialectology, Cambridge, Scholars Publishing, p. 3-26.

BRANDÃo Silvia Figueiredo, 2015, «Concordância nominal em três variedades do português: resultados gerais, novas indagações », Cuadernos de la ALFAL, $\mathrm{n}^{\circ} 7$, p. 36-52.

CAllou Dinah, 1987, Variação e distribuição da vibrante na fala culta carioca, Rio de Janeiro, UFRJ/PROED.

Callou Dinah, 2010, «Quando dialetologia e sociolinguística se encontram », Revista de Estudos Lingüísticos e Literários, vol. 41, p. 30-48.

CAllou Dinah, 2015, «Variação e mudança no âmbito do consonantismo », dans M. A. Martins et J. Abraçado (éds.), Mapeamento sociolingüístico do Português Brasileiro, São Paulo, Contexto.

Callou Dinah \& Brandão Silvia Figueiredo, 2013 (inédit), Caracterização de áreas dialetais no Português do Brasil: análise de duas variáveis.

Callou Dinah, Leite Yonne \& Moraes João Antônio de, 2002, «Processo(s) de enfraquecimento consonantal no português do Brasil», dans M.B.M. Abaurre et A.C.S. Rodrigues (éds), Gramática do português falado, vol. VIII, Novos estudos descritivos, Campinas, UNICAMP/FAPESP, p. 537-555. 
CAllou Dinah \& Marques Maria Helena, 1975, «O -s implosivo na linguagem do Rio de Janeiro », Littera, n $^{\circ}$ 14, p. 9-137.

Callou Dinah \& Moraes João Antônio de, 1995, «Condicionamentos sócio e geolingüísticos na realização do /R/ no português do Brasil », Revista de Estudos Lingüísticos e Literários, vol. 17, p. 69-78.

CARmo Marcia Cristina, 2009, As vogais médias pretônicas dos verbos na fala culta do interior paulista, mémoire de master, Universidade Estadual Paulista, São José do Rio Preto.

Celia Gianni Fontis, 2004, As vogais médias pretônicas na fala culta de Nova Venécia, mémoire de master, Universidade Estadual de Campinas, Campinas.

Chambers Jack K. \& Trudgill Peter, 1980, Dialectology, Cambridge, University Press.

Coelho Izete Lehmkuhl et al., 2015, Para conhecer Sociolinguística, São Paulo, Contexto.

Cunha Celso, 1972, Língua portuguesa e realidade brasileira, Rio de Janeiro, Tempo Brasileiro, $3^{\mathrm{e}}$ éd.

Dias Melina Rezende, 2008, A variação das vogais médias pretônicas no falar dos mineiros de Piranga e Ouro Branco, mémoire de master, Universidade Federal de Minas Gerais, Belo Horizonte.

ElizAicín Adolfo, 2010, «Socio y geolingüística: nueva alianza en los estudios sobre el uso Lingüístico», Revista de Estudos Lingüísticos e Literários, vol. 41, p. 13-28.

Fernández Francisco Moreno, 1996, «Método geolingüístico e método sociolingüístico. El factor "sexo" nos atlas», dans E. Radtke et H. Thun (éds), Neue Wege der romanischen Geolinguistik: Akten des Symposiums zur Empirischen Dialektologie (Heilderberg et Mayence, 21-24 octobre 1991), Kiel, WestenseeVerlag, p. 92-112.

Ferreira Carlota et al., 1987, Atlas Lingüístico de Sergipe, Salvador, UFBA/ FUNDESC.

Gauchat Louis, 1905, L'unité phonétique dans le patois d'une commune, Halle A.D.S., Verlag von Max Niemeyer. Disponible sur <https://archive.org/details/ lunitphontiqued00gaucgoog $>$.

Guy Gregory, 1981, Linguistic Variation in Brazilian Portuguese: Aspects of the Phonology, Syntax, and Language History, PhD Dissertation, University of Pennsylvania, Philadelphie.

GuY Gregory, 1989, «On the Nature and Origins of Vernacular Brazilian Portuguese», dans Estudios sobre el Español de América y Linguística Afroamericana, Bogotá, Instituto Caro y Cuervo, p. 226-244.

HoLm John, 2001-2002, «The Social and Linguistic Origins of Brazilian Vernacular Portuguese: Parallel Cases of Partial Restructuring», Revista Portuguesa de Filologia, vol. 24, p. 123-145.

Kосн Walter et al., 2002, Atlas Lingüístico-etnográfico da Região Sul do Brasil, vol. 1, Porto Alegre, UFRGS ; Florianópolis, UFSC; Curitiba, UFPR.

KuRATH Hans, 1939-1943, Linguistic Atlas of New England, New York, 3 vol. 
LuCCHESI Dante, 2003, «O conceito de transmissão lingüística irregular e o processo de formação do Português do Brasil», dans C. Roncarati et J. Abraçado (éds), Português brasileiro: contato lingüístico, heterogeneidade e história, Rio de Janeiro, 7 Letras, p. 272-284.

Lucchesi Dante, 2012, «A diferenciação da língua portuguesa no Brasil e o contato entre línguas », Estudos de Lingüística Galega, vol. 4, p. 45-65.

Naro Anthony Julius \& Scherre Maria Marta Pereira, 2007, Origens do português brasileiro, São Paulo, Parábola Editorial.

Nascentes Antenor, 1953 [1922], O linguajar carioca, Rio de Janeiro, Organização Simões, $3^{\mathrm{e}}$ éd.

Nobre Wagner Carvalho de Argolo, 2011, Introdução à história das línguas gerais no Brasil: processos distintos de formação no período colonial, mémoire de master, Instituto de Letras, Universidade Federal da Bahia, Salvador.

Mota Jacyra Andrade \& CARdoso Suzana Marcelino, 2015, «Variação fônica nas capitais brasileiras», dans M. A. Martins et J. Abraçado (éds), Mapeamento sociolingüístico do Português Brasileiro, São Paulo, Contexto, p. 65-78.

Mouton Pilar Garcia, 2003, El español de América, Madrid, Consejo Superior de Investigaciones Científicas.

Neri Marcelo Cortes (éd.), 2010, A nova classe média. O lado brilhante dos pobres, Rio de Janeiro, FGV/CPS.

Pickford Glenna, 1956, «American Linguistic Geography: A Sociological Appraisal», Word, no 12, p. 211-233.

Pochmann Márcio, 2012, Nova classe média? O trabalho na base da pirâmide social brasileira, São Paulo, Boitempo.

RADTKe Edgar \& Thun Harald, 1996, «Novos caminos da geolinguística románica. Um balanço », dans E. Radtke et H. Thun (éds), Neue Wege der romanischen Geolinguistik: Akten des Symposiums zur Empirischen Dialektologie (Heidelberg et Mayence, 21-24 octobre 1991), Kiel, Westensee-Verlag, p. 25-49.

Ribeiro José et al., 1977, Esboço de um Atlas Lingüístico de Minas Gerais, Rio de Janeiro, MEC, Casa de Rui Barbosa, UFJF.

Rocha Fabiane de Mello Vianna, 2013, O comportamento das vogais médias pretônicas na fala de Nova Iguaçu, mémoire de master, Universidade Federal do Rio de Janeiro, Rio de Janeiro.

Rocha Fabiane de Mello Vianna \& Brandẽo Silvia Figueiredo, 2014 (inédit), Vogais médias pretônicas na fala da Região Sudeste: um panorama geo-sociolingüístico.

Rossi Nelson, 1963, Atlas Prévio dos Falares Baianos, Rio de Janeiro, MEC/INL.

Silva Neto Serafim da, 1986 [1950], Introdução ao estudo da língua portuguesa no Brasil, Rio de Janeiro, Presença, 5éd.

Thun Harald \& Elizaincín Adolfo, 2000, Atlas Diatópico y Diastrático del Uruguay (ADDU), vol. I, n ${ }^{\text {os }} 1-2$, Kiel, Westensee-Verlag.

Trudgill Peter, 1987, Sociolinguistics: An Introduction to Language and Society, Londres, Penguin Books. 
YACCOUBE Hilaine, 2011, «A chamada "nova classe média”. Cultura material, inclusão e distinção social», Horizontes Antropológicos, vol. 17, no 36, p. 197-231. Yacovenco Lilian Coutinho, 1993, As vogais médias pretônicas no falar culto carioca, mémoire de master, Universidade Federal do Rio de Janeiro, Rio de Janeiro. 